\title{
The accuracy of prediction of genomic selection in elite hybrid rye populations surpasses the accuracy of marker-assisted selection and is equally augmented by multiple field evaluation locations and test years
}

Yu Wang ${ }^{1,2}$, Michael Florian Mette ${ }^{1}$, Thomas Miedaner², Marlen Gottwald ${ }^{3}$, Peer Wilde ${ }^{4}$, Jochen C Reif ${ }^{1 *}$ and Yusheng Zhao ${ }^{1}$

\begin{abstract}
Background: Marker-assisted selection (MAS) and genomic selection (GS) based on genome-wide marker data provide powerful tools to predict the genotypic value of selection material in plant breeding. However, case-to-case optimization of these approaches is required to achieve maximum accuracy of prediction with reasonable input.

Results: Based on extended field evaluation data for grain yield, plant height, starch content and total pentosan content of elite hybrid rye derived from testcrosses involving two bi-parental populations that were genotyped with 1048 molecular markers, we compared the accuracy of prediction of MAS and GS in a cross-validation approach. MAS delivered generally lower and in addition potentially over-estimated accuracies of prediction than GS by ridge regression best linear unbiased prediction (RR-BLUP). The grade of relatedness of the plant material included in the estimation and test sets clearly affected the accuracy of prediction of GS. Within each of the two bi-parental populations, accuracies differed depending on the relatedness of the respective parental lines. Across populations, accuracy increased when both populations contributed to estimation and test set. In contrast, accuracy of prediction based on an estimation set from one population to a test set from the other population was low despite that the two bi-parental segregating populations under scrutiny shared one parental line. Limiting the number of locations or years in field testing reduced the accuracy of prediction of GS equally, supporting the view that to establish robust GS calibration models a sufficient number of test locations is of similar importance as extended testing for more than one year.

Conclusions: In hybrid rye, genomic selection is superior to marker-assisted selection. However, it achieves high accuracies of prediction only for selection candidates closely related to the plant material evaluated in field trials, resulting in a rather pessimistic prognosis for distantly related material. Both, the numbers of evaluation locations and testing years in trials contribute equally to prediction accuracy.
\end{abstract}

Keywords: Marker-assisted selection, Genomic selection, Cross-validation, Hybrid rye, Relatedness, Evaluation locations, Testing years

\footnotetext{
* Correspondence: reif@ipk-gatersleben.de

'Leibniz Institute of Plant Breeding and Crop Plant Research (IPK),

Gatersleben 06466, Germany

Full list of author information is available at the end of the article
} 


\section{Background}

Rye (Secale cereale L.) is an important European crop used for food, feed, and bioenergy that is grown primarily in Eastern, Central and Northern Europe. The main rye belt stretches from the northern parts of Germany through Poland, Ukraine, Belarus, Lithuania and Latvia into central and northern Russia. In contrast to the other major crops of the Triticeae tribe, barley (Hordeum sp.) and wheat (Triticum sp.), rye is an outbreeding species in which selfing is usually prevented by a gametophytic self-incompatibility system [1,2]. As an alternative to open-pollinated varieties, hybrid breeding has been established based on a cytoplasmatic-genic male sterility (CMS) system [3]. Hybrid rye breeding started in 1970 at the University of Hohenheim in Germany and the first hybrid varieties were released in Germany in 1984 [4]. Economically important traits in hybrid rye are, among others, grain yield and plant height in context of productivity as well as starch content and total pentosan content with regard to end user quality [5].

Current plant breeding programs are making extensive use of molecular markers to predict the performance potential of the involved plant material. In particular, marker-assisted selection (MAS) is widely applied, but is not necessarily an optimal approach for complex agronomic traits as it is usually based on predictions derived from only a few markers in linkage disequilibrium (LD) to large effect quantitative trait loci (QTL) and, thus, ignores the contributions from small to intermediate effect QTL $[6,7]$. To advance the accuracy of prediction, genomic selection (GS) has been suggested as an extension of MAS [8]. In GS, a large number of molecular markers distributed evenly over the genome is used to train the prediction model. Sufficient marker density provided, GS potentially makes use of all the genetic variance present in an analyzed population by summing the effects of all individual markers [7] and thus can be expected to also include information from small effect gene loci that cannot be captured by traditional QTL determination via MAS [9]. Accordingly, GS is of growing importance for efficient and cost-effective breeding programs [8].

In this context, cross-validation approaches have become an important tool for the empirical evaluation of the accuracy of prediction. Here, a population of plants for which phenotyping and high density genotyping data are available is split into two subsets, an estimation set and a test set. Marker effects are then determined based on the data from the estimation set, followed by the prediction of the genotypic values of the plants in the test set based on the estimated marker effects. The correlation of predicted and observed values in the test set provides a measure for the accuracy of prediction. This has been applied to e.g. test-cross populations of maize [10-13], wheat $[14,15]$ and barley $[16,17]$.
Several analytical approaches based on different assumptions with regard to the marker effects have been proposed for GS $[8,18]$. Bayesian methods such as Bayes A estimate the variances of the effects of markers separately, while ridge regression best linear unbiased prediction (RR-BLUP) assumes that all marker effects are normally distributed and have identical variance [8]. RR-BLUP has been proved to afford high prediction accuracies across crops and traits [16] and is suitable for GS of complex traits $[19,20]$.

In two bi-parental segregating populations used in test-crosses to produce hybrid rye, grain yield, plant height, starch content, and total pentosan content were reported to represent quantitative traits controlled by multiple small to medium effect QTL [5]. Using data sets from this material, we show in our current study that GS has a consistently superior accuracy of prediction in comparison to MAS. Relatedness of the plant material included in the estimation and test sets clearly affects the accuracy of prediction, and limiting the number of locations in field testing has almost the same impact than limiting the number of years. This supports the view that establishing calibration models for GS requires phenotyping across locations and years.

\section{Methods}

\section{Plant material and field experiments}

The plant materials and field experiments used to obtain the data sets analyzed in this study are described in detail in Miedaner et al. [5]. In brief, three elite winter rye inbred lines (Lo90-N, Lo115-N and Lo117-N) were used as parents to generate segregating population $\mathrm{A}$ (Pop-A, Lo115-N $\times$ Lo90-N) and population B (Pop-B, Lo115-N $\times$ Lo117-N), respectively. $F_{1}$ plants from crosses of parental lines were self-pollinated under isolation bags during two generations to obtain $\mathrm{F}_{3}$ plants by single seed descent. From each population, Pop-A and Pop-B, 220 randomly selected $\mathrm{F}_{3}$ progenies were used for pollination of a cytoplasmically male sterile (CMS) single cross tester $(\mathrm{X} \times \mathrm{Y})$ between isolation walls resulting in three-way hybrids of the type $(\mathrm{X} \bullet \mathrm{Y}) \times \mathrm{F}_{3: 4}$. The CMS tester was genetically unrelated to the parents of both populations.

Field experiments with these hybrids were carried out in two years (2010 and 2011) at five locations, Wohlde (WOH, Germany, N52.8 $8^{\circ}$ E10.0 ${ }^{\circ}, 80 \mathrm{~m}$ above sea level), Beckedorf (BEK, Germany, N52.5 ${ }^{\circ}, \mathrm{E} 10.3^{\circ}, 80 \mathrm{~m}$ above sea level), Petkus (PET, Germany, N51.6 ${ }^{\circ}$ E13.2 ${ }^{\circ}, 130 \mathrm{~m}$ above sea level), Stuttgart/Hohenheim $\left(\mathrm{HOH}, \mathrm{N} 48.4^{\circ}\right.$, $\mathrm{E} 9.1^{\circ}, 400 \mathrm{~m}$ above sea level), and Walewice (WAL, Poland, N52.6 ${ }^{\circ}, \mathrm{E} 19.4^{\circ}, 184 \mathrm{~m}$ above sea level). The respective location $\times$ year combinations are denoted as environments WOH10, WOH11, BEK10, BEK11, PET10, PET11, HOH10, HOH11, WAL10 and WAL11 throughout this study. 
Hybrid collections derived from segregating $\mathrm{F}_{3: 4}$ lines from both populations were evaluated together with hybrids obtained from their parental lines (repeated 9 times) as well as 2 common checks in field traits using an incomplete $24 \times 10$ alpha design with two replications. Data for grain yield $\left(\mathrm{dt} \mathrm{ha}{ }^{-1}\right)$, plant height $(\mathrm{cm})$, starch content (\%) and total pentosan content (\%) of hybrids were obtained as described by Miedaner et al. [5]. Starch content (\%) and total pentosan content (\%) were determined by near-infrared reflectance spectroscopy (NIRS) recorded with a Bruker MPA FT- NIRS instrument (Bruker Optics Ettligen) in reflectance mode over the range from 850 to $2500 \mathrm{~nm}$. The samples were scanned twice in duplicate repacking using two different Petri dishes of $8.7 \mathrm{~cm}$ diameter as sampling cups on a rotating device with on average 32 scans in 10 seconds. Prediction models were calculated with OPUS Software version 6.5 (Bruker Optics Ettligen). Calculations were carried out with a modified partial least squares (PLS) procedure using a 1st derivation and a scatter correction (SNV) of the spectra. Samples were randomly assigned to calibration and validation sets, and calibration was performed based on chemical quantification methods [21]. Finally, the suitability of the models was controlled within the validation set. For grain yield and plant height, data across nine environments (BEK10, BEK11, PET10, PET11, HOH10, WAL10, WAL11, WOH10, and WOH11) were included in our analysis. Data from HOH11 were not used due to low repeatability (0.01 in Pop-A and 0.00 in Pop-B for grain yield and 0.69 in Pop-A and 0.19 in Pop-B for plant height, respectively). For starch content and total pentosan content, data across six environments (PET10, PET11, WAL10, WAL11, WOH10, and WOH11) were included.

\section{Phenotypic data analysis}

Best linear unbiased estimates (BLUEs) for testcross progenies across environments were determined by the restricted maximum likelihood method using ASReml version 3.0 [22] based on a two-step linear regression model:

$$
\begin{aligned}
& \text { Step } 1 \\
& \qquad y_{E n v}=1_{n} \mu+G \alpha_{G}+R \alpha_{R}+B \alpha_{B}+e
\end{aligned}
$$

where $y_{\text {Env }}$ refers to the BLUEs of each plot, $1_{n}$ is a vector with the length $\mathrm{n}$ equal to the number of genotypes times the number of replications, $\mu$ denotes the overall mean, $G$ is a design matrix for fixed effects of the genotypes, $\alpha_{\mathrm{G}}$ refers to a $\mathrm{N}$-vector of the genotype effects with $\mathrm{N}$ equal to the number of genotypes, $R$ is a design matrix for random effects of the replication, $\alpha_{R}$ represents a vector of the replication effects, $B$ is a design matrix for random effects of the block, $\alpha_{\mathrm{B}}$ refers to a vector of the block effects and $e$ is a residual term. With step 1, BLUEs of testcross progenies within each environment were estimated, which were then applied in step 2 to estimate BLUEs of testcross progenies across nine or six environments, respectively.

$$
\begin{aligned}
& \text { Step } 2 \\
& \qquad y=1_{\mathrm{k}} \mu+G \alpha_{G}+E \alpha_{\mathrm{Env}}+F \alpha_{\mathrm{F}}+e
\end{aligned}
$$

where $y$ refers to the BLUEs across all the environments, $1_{\mathrm{k}}$ is a vector with the length $\mathrm{k}$ equal to the number of genotypes times the number of environments, $E$ is a design matrix assigning random environment effects to the phenotypes, $\alpha_{\mathrm{Env}}$ is a vector of environments effects, $F$ denotes a design matrix of random interaction effects of genotype $\times$ environment, $\alpha_{\mathrm{F}}$ is a vector of interaction effects and $e$ is a residual term.

The same linear regression model, in which in both, step 1 and step 2, G can be viewed as a design matrix for random effects of the genotypes, was applied to estimate the variance components, including genotypic variance $\left(\sigma_{G}^{2}\right)$, genotype $\times$ environment interaction vari-

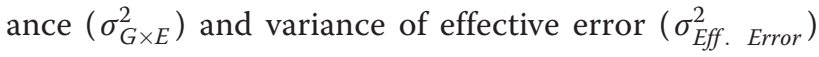
across both segregating populations (Table 1). Heritability $\left(h^{2}\right)$ was estimated as $h^{2}=\frac{\sigma_{G}^{2}}{\sigma_{G}^{2}+\frac{\sigma_{G \times E}^{2}}{N r \cdot E n v}+\frac{\sigma_{E f f . E r r o r}^{2}}{N r \cdot R e p \times N r \cdot E n v} .}$, where $\sigma_{G}^{2}$ is the genotypic variance across nine (for grain yield and plant height) or six (for starch content and total pentosan content) environments, respectively. Nr.Env. and Nr.Rep. refer to the number of environments and replications, separately, and $\sigma_{E f f}^{2}$. Error denotes the variance of effective error across nine or six environments, respectively [23]. The broad-sense heritability of each environment, denoted as repeatability $(r)$, was calculated as $r=\frac{\sigma_{G}^{2}}{\sigma_{G}^{2}+\frac{\sigma_{E f f . E r r o r}^{2}}{N r \cdot \text { Rep }}}$, where $\sigma_{G}^{2}$ and $\sigma_{\text {Eff. Error }}^{2}$ are the genotypic variance and the variance of effective error within each environment, respectively [23].

\section{Genotypic data analysis}

Each of the two times $220 \mathrm{~F}_{3: 4}$ lines in population $\mathrm{A}$ and population $B$ had been genotyped with simple sequence repeat (SSR), single nucleotide polymorphism (SNP) and diversity array technology (DArT) markers [5]. We reapplied quality checks to these marker data, excluding markers with (i) a rate of missing values above $5 \%$ and (ii) allele frequencies smaller than 0.05 or larger than 0.95 , and complemented missing genotypes according to a binomial distribution. If not indicated otherwise, only data from DArT markers (394 for population A, 584 for 
Table 1 Estimates of variance components and heritability $\left(h^{2}\right)$ for grain yield, plant height, starch content and total pentosan content among 220 test-cross progenies each, obtained using $F_{3: 4}$ from two bi-parental segregating populations, population A and population B

\begin{tabular}{|c|c|c|c|c|c|c|}
\hline Traits & Mean & Range & $\sigma_{G}^{2}$ & $\sigma_{G \times E}^{2}$ & $\sigma_{\text {Eff.Error }}^{2}$ & $h^{2}$ \\
\hline & \multicolumn{6}{|c|}{ Population A } \\
\hline Grain yield & 79.3 & $73.4-85.4$ & $3.33^{* *}$ & $8.40^{* *}$ & 3.48 & 0.75 \\
\hline Plant height & 118.2 & $110.9-126.6$ & $7.30^{* *}$ & $4.03^{* *}$ & 2.94 & 0.92 \\
\hline Starch content & 61.6 & $60.0-62.9$ & $0.24^{* *}$ & $0.26^{* *}$ & 0.14 & 0.87 \\
\hline \multirow[t]{2}{*}{ Total pentosan content } & 10.0 & $9.5-10.6$ & $0.03^{* *}$ & $0.08^{* *}$ & 0.04 & 0.75 \\
\hline & \multicolumn{6}{|c|}{ Population B } \\
\hline Grain yield & 75.6 & $69.5-83.4$ & $3.76^{* *}$ & $9.18^{* *}$ & 3.72 & 0.75 \\
\hline Plant height & 115.6 & $104.5-127.4$ & $12.62^{* *}$ & $4.02^{* *}$ & 2.22 & 0.96 \\
\hline Starch content & 61.5 & $59.8-63.4$ & $0.44^{* *}$ & $0.24^{* *}$ & 0.14 & 0.93 \\
\hline Total pentosan content & 10.3 & $9.6-11.0$ & $0.03^{* *}$ & $0.09^{* *}$ & 0.04 & 0.73 \\
\hline
\end{tabular}

$\sigma_{G}^{2}$ refers to the genotypic variance, $\sigma_{G \times E}^{2}$ represents the interaction variance between genotype and environment,and $\sigma_{E f f . E r r o r}^{2}$ denotes the variance of effective error.

** Significantly different from zero with $P<0.01$.

population B, and 1048 for combined populations A and B) were included in analysis. Linkage disequilibrium (LD) was estimated using the squared allele frequency correlations $\left(r^{2}\right)$ [24]. The LD structures in population $\mathrm{A}$, population $\mathrm{B}$, and combined populations $\mathrm{A}$ and $\mathrm{B}$ are provided in Additional file 1: Figure S1.

\section{Marker-assisted selection}

Marker-assisted selection based on QTL (MAS-QTL) was performed within population B in context with QTL mapping via PLABQTL [25] employing composite interval mapping (CIM) by the regression approach [26] in combination with the use of cofactors $[27,28]$. We contrasted MAS based on the detected QTL, with MAS based on a random sample of molecular markers. This "neutral marker-assisted selection" (MAS-NEUT) uses markers that were randomly selected according to the number of QTL identified with the respective limit of detection (LOD) score in MAS-QTL for the corresponding trait (first one from each of the seven linkage groups in rye, then randomly chosen additional ones in the case of more than seven QTL). For MAS-QTL, crossvalidation was implemented within PLABQTL [25] and accuracies of prediction were calculated as $r_{g}=\sqrt{\frac{R_{C V}^{2}}{h^{2}}}$, where $R_{C V}^{2}$ denotes the percentage of phenotypic variance the test set explained by identified QTL and $h^{2}$ represents heritability [5]. For MAS-NEUT, cross-validation was implemented within population $B$ according to scheme $\mathrm{CV}_{\mathrm{G}}$ Within-Within as described below.

\section{Genomic selection}

Breeding values were estimated by model, $y=\mu 1_{N}+X a$ $+e$, where $y$ is an $N \times 1$ vector of BLUEs estimated across environments; $\mu$ represents overall mean, $1_{N}$ refers to a vector with the length $N, a$ is the marker effect, $X$ refers to a design matrix for the marker effect, and $e$ denotes a residual. By using ridge regression best linear unbiased prediction (RR-BLUP) [29], the estimated marker effects $(\hat{a})$ were estimated based on a mixed model equation, $\left[\begin{array}{cc}1_{N}^{T} 1_{N} & 1_{N}^{T} X \\ X^{T} 1_{N} & X^{T} X+I \lambda\end{array}\right]\left[\begin{array}{c}\hat{\mu} \\ \hat{a}\end{array}\right]=\left[\begin{array}{c}1_{N}^{T} y \\ X^{T} y\end{array}\right]$, where $1_{N}^{T}$ is the transpose of $1_{N}, X^{\mathrm{T}}$ represents the transpose of $X, I$ is an identity matrix, $\lambda$ represents a penalty parameter, and $\hat{\mu}$ denotes the estimated overall mean. The penalty parameter can be calculated as $\lambda=m\left(\frac{1}{h^{2}}-1\right)$, where $m$ is the number of markers and $h^{2}$ refers to the heritability of the estimation set [30]. Then the genetic values were predicted as $\mathrm{PV}=X \hat{a}$, where $\hat{a}$ is the estimated marker effect.

\section{Cross-validation}

In all cross-validation approaches, data sets were divided into an estimation set (ES) that was used to estimate marker effects, and a test set (TS), in which the predictive ability (Pearson correlation $r_{\mathrm{MP}}$ ) between observed BLUEs and the genotypic values predicted based on the determined marker effects was calculated to provide a measure of the accuracy of prediction [11]. Correlations were either determined as accuracy of prediction $r_{\mathrm{p}}=r_{\mathrm{MP}}$ or as standardized accuracy of prediction $r_{\mathrm{g}}=r_{\mathrm{MP}} / h$ calibrated by the square root of heritability $[10,31,32]$. Sampling of estimation and test sets was repeated 5,000 times in each cross-validation scheme.

For cross-validation across genotype $\left(\mathrm{CV}_{\mathrm{G}}\right)$, data sets were split into five equally sized subsets (S1 to S5). Four subsets (S1-S4) comprised the ES for estimating marker effects, while the remaining subset (S5) served as TS. Members of the ES and TS were taken either from 
individual populations $\left(\mathrm{CV}_{\mathrm{G}}\right.$ Within-Within) from both populations $\left(\mathrm{CV}_{\mathrm{G}}\right.$ Across-Across), or the ES was taken from both populations, population $\mathrm{A}$ and $\mathrm{B}$, while the TS was taken from one population $\left(\mathrm{CV}_{\mathrm{G}}\right.$ Across-Within). In the case that ES and TS were from individual populations $\left(\mathrm{CV}_{\mathrm{G}}\right.$ Within-Within) they could either originate from the same population $\left(\mathrm{CV}_{\mathrm{G}}\right.$ Within-Within-Same) or from different populations $\left(\mathrm{CV}_{\mathrm{G}}\right.$ Within-Within-Different).

Cross-validation across genotype based on different numbers of environments $\left(\mathrm{CV}_{\mathrm{G}}\right.$ Env) was conducted separately within population $\mathrm{A}$ and population $\mathrm{B}\left(\mathrm{CV}_{\mathrm{G}}\right.$ Within-Within-Same). Data for the ES and TS were taken from one to nine randomly permutated locationyear combinations. Cross-validation across genotype within location $\left[\mathrm{CV}_{\mathrm{G}(\mathrm{L})}\right]$, across genotype and location $\left(\mathrm{CV}_{\mathrm{G} \times \mathrm{L}}\right)$, across genotype within year $\left[\mathrm{CV}_{\mathrm{G}(\mathrm{Y})}\right]$ and across genotype and year $\left(\mathrm{CV}_{\mathrm{G} \times \mathrm{Y}}\right)$ was also implemented separately within population $\mathrm{A}$ and population $\mathrm{B}\left(\mathrm{CV}_{\mathrm{G}}\right.$ Within-Within-Same). For cross-validation across genotype and within location $\left[\mathrm{CV}_{\mathrm{G}(\mathrm{L})}\right]$ or across genotype and location $\left(\mathrm{CV}_{\mathrm{G} \times \mathrm{L}}\right)$, data for the ES were derived from two randomly selected locations over the two years 2010 and 2011 (e.g. BEK10, BEK11, PET10, and PET11), while the data for the TS were taken either from the same location-year combinations $\left[\mathrm{CV}_{\mathrm{G}(\mathrm{L})}\right]$ (e.g. BEK10, BEK11, PET10, and PET11), or from the remaining four locationyear combinations $\left(\mathrm{CV}_{\mathrm{G} \times \mathrm{L}}\right)$ (e.g. WAL10, WAL11, PET10, and PET11, but always excluding HOH10). In contrast, for cross-validation across genotype and within year $\left[\mathrm{CV}_{\mathrm{G}(\mathrm{Y})}\right]$ or across genotype and year $\left(\mathrm{CV}_{\mathrm{G} \times \mathrm{Y}}\right)$, data for the ES were collected from one year, either 2010 or 2011, at four locations (e.g. BEK10, PET10, WAL10, and WOH10), with data for the TS taken either from the same year $\left[\mathrm{CV}_{\mathrm{G}(\mathrm{Y})}\right]$ (e.g. BEK10, PET10, WAL10, and WOH10, but always excluding $\mathrm{HOH} 10$ ) or from the other year $\left(\mathrm{CV}_{\mathrm{G} \times \mathrm{Y}}\right)$ (e.g. BEK11, PET11, WAL11, and WOH11).

\section{Results}

Field trials and genotyping analysis

Field trials with hybrid rye from test-crosses involving $220 \mathrm{~F}_{3: 4}$ members and their two parental elite inbred lines of bi-parental segregating population A and population B, respectively, were performed at five locations in Germany and Poland in the years 2010 and 2011 as reported in detail by Miedaner et al. [5]. High quality evaluation data with heritabilities in the range from 0.73 to 0.96 (Table 1) were obtained from nine location-year combinations for grain yield and plant height and from six location-year combinations for starch content and total pentosan content. Populations A and B were derived from crosses Lo115-N $\times$ Lo90-N and Lo115-N $\times$ Lo117-N, respectively, sharing one common parent, Lo115-N. Thus, their members can be considered as half-sibs. Both populations were characterized by the presence of broad genotypic variance $\sigma_{G}^{2}$ as well as interaction variance between genotype and environment $\sigma_{G \times E}^{2}$ for grain yield, plant height, and starch content, and, to a lesser extent, total pentosan content (Table 1). For all traits analyzed, significant $(P<0.01)$ genotypic variance and variation due to genotype $\times$ environment interaction effects was obtained in both populations. Consistent with this, genotypic values for the four traits covered broad ranges (Additional file 2: Figure S2). Medians for all traits differed between the two populations, in particular with regard to grain yield, but genotypic variations indicated by the $50 \%$-quartile were quite similar. Except for grain yield, genotypic values of the parents of both populations were rather close to the median. Genotyping based on 1048 molecular markers revealed 394 markers segregating among members of population A and 584 markers segregating among members of population $B$.

\section{Standarized accuracy of prediction of marker-assisted selection in comparison to genomic selection}

Cross-validated accuracies of prediction based on markerassisted selection (MAS) for grain yield, plant height, starch content, and total pentosan content in hybrid rye from test-crosses involving the two segregating populations have been reported previously [5]. Performing crossvalidated genomic selection (GS) using RR-BLUP [29] based on the very same dataset, we found consistently higher standardized accuracies of prediction in both populations for all four traits that were analyzed (Figure 1). In particular, GS increased the standardized accuracy of prediction from 0.12 with MAS to 0.59 for grain yield in population A and from 0.28 with MAS to 0.70 for total pentosan content in population B. Comparable, albeit less drastic, increases of accuracy were obtained with GS for all other traits in both populations. In order to further explore the potential limitations of MAS, the standardized accuracies of prediction by MAS based on mapped QTL (MAS-QTL) for population B were compared to the results of neutral marker-assisted selection (MAS-NEUT) performed based on randomly selected markers distributed equally across linkage groups over a range of limit of detection (LOD) values, which also generated substantial standardized accuracies of prediction (Additional file 3: Figure S3). Thus, taking into account the consistently lower standardized accuracies of predictions of MAS in combination with the potential over-estimation, all further analysis was based on GS using RR-BLUP.

\section{Accuracy of prediction of genomic selection within and across populations}

Taking advantage of the unique design of the two biparental segregating populations sharing one parental 

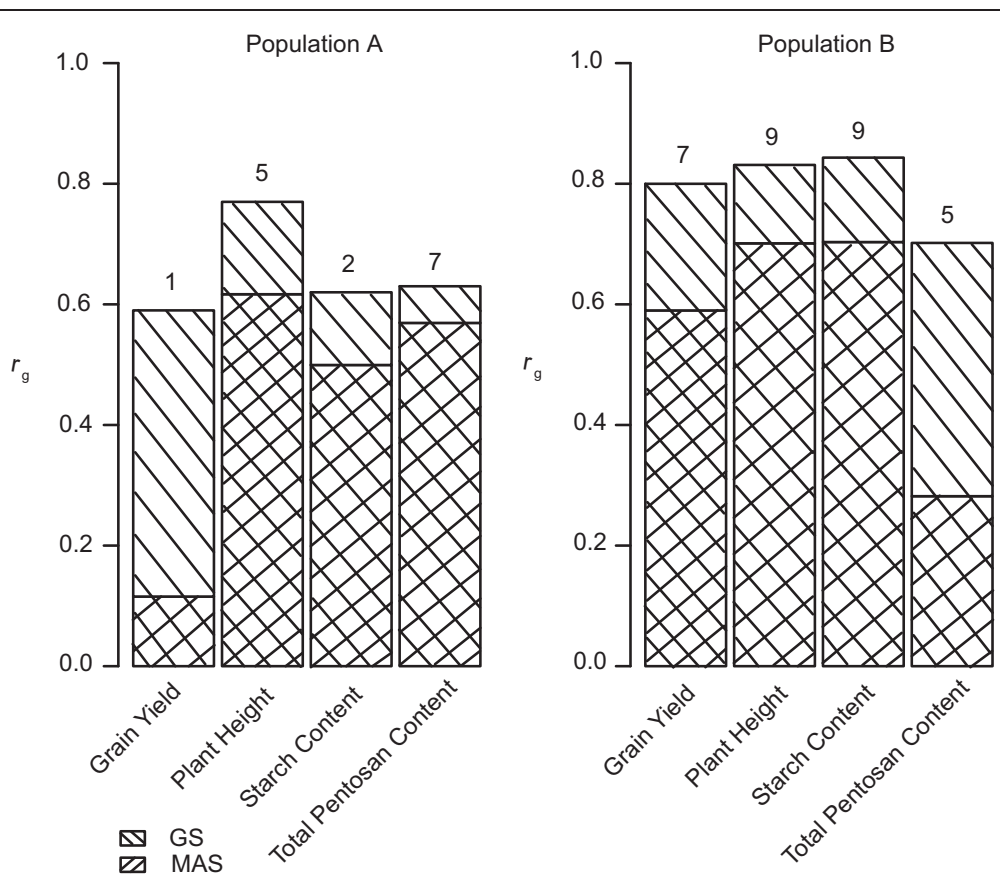

Figure 1 Cross-validated standardized accuracies of prediction $\left(r_{g}\right)$ for genomic selection compared to marker-assisted selection.

Genomic selection (GS) was based on ridge regression best linear unbiased prediction (RR-BLUP), while marker-assisted selection (MAS) was based on QTL mapped with a limit of detection (LOD) threshold of 3.73 and the detected QTL (numbers above columns). Cross-validation was performed separately within population $\mathbf{A}$ and population $\mathbf{B}\left(\mathrm{CV}_{\mathrm{G}}\right.$ Within-Within-Same) for traits grain yield, plant height, starch content, and total pentosan content. Accuracies of prediction for MAS were taken from [5] and were based on SSR and DArT markers for population $\mathbf{A}$ and on SSR and SNP markers for population $\mathbf{B}$

inbred line in common, we applied $\mathrm{CV}_{\mathrm{G}}$ with different estimation set-test set combinations in order to study the dependency of the accuracy of prediction of GS on the relatedness of the included material (Figure 2). Separate cross-validation across genotype among sibs only within each population $\left(\mathrm{CV}_{\mathrm{G}}\right.$ Within-WithinSame; Figure 2, category I) showed consistently lower accuracies of prediction for population A than population B for all four traits. For example, the median accuracy of prediction $r_{p}$ was approximately 0.51 for population A and 0.70 for population $B$ for grain yield and approximately 0.75 for population $\mathrm{A}$ and 0.82 for population B for plant height, respectively. Crossvalidation across genotype among combined sibs and half-sibs with estimation and test sets taken from both populations $\left(\mathrm{CV}_{\mathrm{G}}\right.$ Across-Across; Figure 2, category II) generated a slight increase of the accuracies of prediction in the cases of grain yield and total pentosan content, but accuracies of prediction for the other two traits were approximately intermediate between the accuracies of prediction for sibs within populations $\mathrm{A}$ and $\mathrm{B}$, respectively, in $\mathrm{CV}_{\mathrm{G}}$ Within-Within-Same. When the estimation set was extended across the two populations to include sibs and half-sibs, but the test set was restricted to sibs from one population only $\left(\mathrm{CV}_{\mathrm{G}}\right.$
Across-Within; Figure 2, category III), the accuracies of prediction were essentially the same as when estimation set and test set were from sibs from the same population in $\mathrm{CV}_{\mathrm{G}}$ Within-Within-Same. Finally, when the estimation set was taken from within one population and the test set from the other population $\left(\mathrm{CV}_{\mathrm{G}}\right.$ Within-Within-Other; Figure 2, category IV) for prediction among half-sibs only, accuracies of prediction were substantially lower than in the $\mathrm{CV}_{\mathrm{G}}$ Within-Within-Same scenario among sibs for all traits analyzed.

\section{Accuracy of prediction of genomic selection across genotypes based on different numbers of location-year combinations}

In order to judge the contribution of the extension of field trials to the accuracy of prediction of genomic selection, cross-validation across genotype was performed separately within population A and population B for grain yield and plant height based on increasing numbers of environments, that is, location-year combinations $\left(\mathrm{CV}_{\mathrm{G}}\right.$ Env; Figure 3). Accuracies of prediction were consistently lower for population A in comparison to population B. They increased continuously with the number of included environments, with the gain per added environment being lower for grain yield and higher for plant height. 


\section{Accuracy of prediction of genomic selection within or across locations and years}

The availability of data sets from five locations over two years for grain yield and plant height allowed us to estimate the effects of limiting the number of locations or number of years on accuracies of prediction, which is pivotal for the optimal allocation of resources in field trials. Analysis was done separately within either population A or population B based on estimation sets from four location-year combinations. Test sets were taken from the same four location-year combinations $\left[\mathrm{CV}_{\mathrm{G}(\mathrm{L})}\right.$ and $\mathrm{CV}_{\mathrm{G}(\mathrm{Y})}$; Figure 4], or from the remaining four available location-year combinations excluding $\mathrm{HOH} 10\left(\mathrm{CV}_{\mathrm{G} \times \mathrm{L}}\right.$ and $\mathrm{CV}_{\mathrm{G} \times \mathrm{Y}}$; Figure 4). The accuracy of prediction of GS across genotype within location $\left[\mathrm{CV}_{\mathrm{G}(\mathrm{L})}\right]$ or across genotype and location $\left(\mathrm{CV}_{\mathrm{G} \times \mathrm{L}}\right)$ was determined by selecting data from two locations in the years 2010 and 2011 for estimation, the accuracy of prediction of GS across genotype within year $\left(\mathrm{CV}_{\mathrm{G}(\mathrm{Y})}\right)$ or across genotype and year $\left(\mathrm{CV}_{\mathrm{G} \times \mathrm{Y}}\right)$ was derived from estimation based on data from four locations in 2010 or 2011. Accuracies of prediction in $\mathrm{CV}_{\mathrm{G}(\mathrm{L})}$ were approximately the same as in

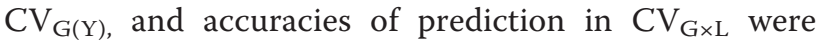
nearly the same as in $C V_{\mathrm{G} \times \mathrm{Y}}$ in both populations for 

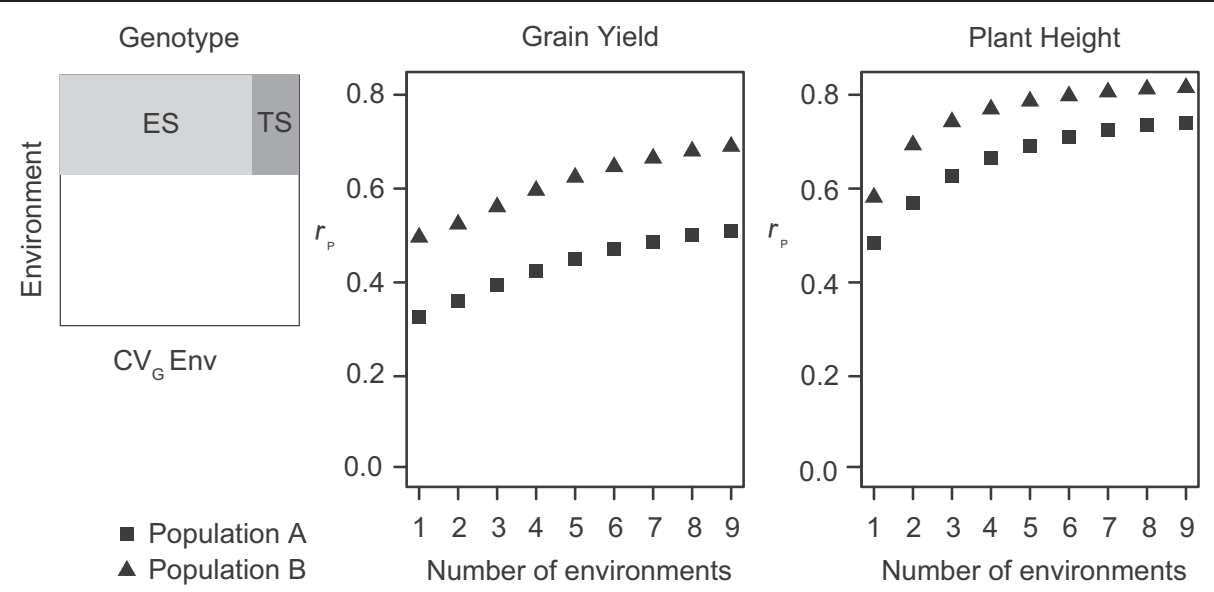

Figure 3 Cross-validated accuracies of prediction for genomic selection using increasing environment numbers (CV $\mathrm{G}_{\mathrm{G}}$ Env). Calculation was done with RR-BLUP across genotype. ES in the scheme at the left refers to the estimation set, TS represents the test set. At the right, the impact of increasing the number of environments used for cross-validation on the accuracy of prediction $r_{p}$ for grain yield and plant height in population $\mathbf{A}$ (squares) and population $\mathbf{B}$ (triangles).

each of the analyzed traits. When estimation and test sets were collected from the different location-year combinations $\left(\mathrm{CV}_{\mathrm{G} \times \mathrm{L}}\right.$ and $\left.\mathrm{CV}_{\mathrm{G} \times \mathrm{Y}}\right)$, accuracies of prediction were consistently lower in comparison to the situation with both estimation and test sets form the same location-year combinations $\left[\mathrm{CV}_{\mathrm{G}(\mathrm{L})}\right.$ and $\left.\mathrm{CV}_{\mathrm{G}(\mathrm{Y})}\right]$.

\section{Discussion}

The superior standardized accuracy of prediction of GS in comparison to MAS for the complex traits grain yield, plant height, starch content, and total pentosan content in rye (Figure 1) is in line with observations for diverse traits in other crops $[33,34]$. The limitations of MAS in comparison to GS have been discussed in detail previously [7-9,35]. In the context of our study, the analysis of MAS-NEUT based on randomly selected markers revealed a substantial contribution to the standardized accuracy of prediction that is not dependent on informative markers linked to QTL (Additional file 3: Figure S3). With MAS-NEUT representing a kind of special case of genomic selection, this might be due to the relatedness of genotypes in the respective population [18, 36-38].

Focusing on genomic selection via RR-BLUP, we explored accuracies of prediction within and across biparental populations as well as across field trial locations and years. The accuracies of prediction for populationspecific test-sets were rather similar, no matter whether estimation was done within $\left(\mathrm{CV}_{\mathrm{G}}\right.$ Within-Within-Same) or across populations $\left(\mathrm{CV}_{\mathrm{G}}\right.$ Across-Within) (Figure 2). $A$ reason for this could have been the close relationship of population $\mathrm{A}$ and population $\mathrm{B}$, being half-sibs sharing one parental line (Lo115-N). However, accuracies of prediction were consistently higher for test-sets from population $B$. This cannot be explained by variation in the quality of field trials, as heritabilities seen with hybrids derived from population $\mathrm{A}$ and population B were similar for all four traits considered (Table 1). A possible explanation for the higher accuracy would be the presence of higher genetic diversity in population B in comparison to population A. This view is supported by the higher genetic variance $\sigma_{G}^{2}$ in population $B$ for three of the four traits in study as well as the higher number of segregating molecular markers (584 versus 394). Consistently, population A was generated by crossing two superior test-cross lines, Lo115$\mathrm{N}$ and Lo90-N, while population B was obtained by crossing one superior line, Lo115-N with a lower performing line, Lo117-N [5]. A similar positive effect of higher genetic variation on the accuracy of prediction in genomic selection has been reported by Zhao et al. [39] and Riedelsheimer et al. [40]. However, also the higher number of polymorphic markers in population $\mathrm{B}$ per se might contribute to higher accuracy of prediction [41]. Actually, the rather low number of markers used in analysis might present a general limitation of the accuracy of genomic selection in our current study. This limitation could be overcome by taking advantage of recently developed SNP arrays for rye [42]. When genomic selection was done solely across populations $\left(\mathrm{CV}_{\mathrm{G}}\right.$ Across-Across), either a slight decrease or a slight increase of the accuracy of prediction was observed depending on the trait. This could be due to the difference among the two populations. For grain yield and total pentosan content, accuracies of prediction were higher in $\mathrm{CV}_{\mathrm{G}}$ (Across-Across) than in $\mathrm{CV}_{\mathrm{G}}$ (Within-Within-Same) and $\mathrm{CV}_{\mathrm{G}}$ (Across-Within). This could be caused by a larger genetic diversity among populations in comparison to the genetic diversity within each population. In contrast, for plant height, 
the genetic variation among two populations was not quite large, and for starch content the genetic variation among two populations was rather small, leading to accuracies of prediction in $\mathrm{CV}_{\mathrm{G}}$ (Across-Across) between the accuracies of prediction of $\mathrm{CV}_{\mathrm{G}}$ (WithinWithin-Same) and $\mathrm{CV}_{\mathrm{G}}$ (Across-Within). When genomic selection was attempted from one population to the other $\left(\mathrm{CV}_{\mathrm{G}}\right.$ Within-Within-Different), accuracies 
of prediction were consistently lower than in all other approaches, readily revealing the limits of predicting among half-sib populations in hybrid rye. This is consistent with the in general rather pessimistic prognosis for GS-based prediction for material distantly related to the plants evaluated in field trials in diverse crops $[40,43,44]$.

With the continuous reduction of genotyping costs over time, phenotypic evaluation in field trials has now become the more cost-intensive action in the calibration of MAS or GS in plant breeding programs [45,46]. As there is consistent pressure to reduce costs, reduction of field trial expenses is a tempting option. In addition, the established phenotypic selection schemes usually allow only one year of field testing for the vast majority of selection candidates. However, cross-validation testing of the impact of field trial size on the accuracy of prediction of genomic selection $\left(\mathrm{CV}_{\mathrm{G}} \mathrm{Env}\right)$ as indicated by $r_{\mathrm{p}}$ in our study revealed a clear dependence on the number of location-year combinations for hybrid rye (Figure 3). The grade of this dependence varied for the analyzed traits in hybrid rye. In the case of plant height, increases of accuracy were only marginal beyond the inclusion of data from five environments, while in the case of grain yield, the accuracy was still substantially increasing when all available data from nine environments were included, underlining the need for testing over a sufficient number of location-year combinations for optimal calibration. An explanation for the difference between the two traits might lie in the lower heritability seen for grain yield in comparison to plant height (Table 1).

In order to separately check the specific impacts of the number of field trial locations and the number of test years on the prediction accuracy, we performed crossvalidated genomic selection across genotype within location $\left[\mathrm{CV}_{\mathrm{G}(\mathrm{L})}\right]$, or across genotype and location $\left(\mathrm{CV}_{\mathrm{G} \times \mathrm{L}}\right)$ using data from two out of four locations for estimation, and across genotype within year $\left[\mathrm{CV}_{\mathrm{G}}(\mathrm{Y})\right]$, or across genotype and year $\left(\mathrm{CV}_{\mathrm{G} \times \mathrm{Y}}\right)$ using data from one out of two years (Figure 4). The accuracies of prediction were consistently higher in $\mathrm{CV}_{\mathrm{G}(\mathrm{L})}$ and $\mathrm{CV}_{\mathrm{G}(\mathrm{Y})}$ with test sets from the same subset of locations or the same year, respectively, than in $\mathrm{CV}_{\mathrm{G} \times \mathrm{L}}$ and $\mathrm{CV}_{\mathrm{G} \times \mathrm{Y}}$, with the test set taken from the locations or the year not included in the estimation set. Thus, the accuracy of prediction was limited by genotype and location and genotype and year interactions. A similar limitation from location to location prediction accuracy has been reported for MAS in hybrid maize [11]. According to Ly et al. [44], the "overestimation" of the accuracy of prediction resulting from taking estimation and test sets from the same environments can be determined based on the magnitude of genotype and environment interaction effect
$\left(\mathrm{G} \times \mathrm{E}\right.$ interaction) $\frac{\sigma_{G \times E}^{2}}{\sigma_{G}^{2}+\sigma_{G \times E}^{2}}$. It varies substantially for different traits. Based on the $\sigma_{G}^{2}$ and $\sigma_{G \times E}^{2}$ values from Table 1, the ratio of $\mathrm{G} \times \mathrm{E}$ interaction for grain yield was 0.72 and 0.71 in population $A$ and population $B$, respectively, compared to a ratio of $\mathrm{G} \times \mathrm{E}$ interaction for plant height of 0.36 and 0.24 in population $A$ and population $B$ in our study. The larger ratio of $\mathrm{G} \times \mathrm{E}$ interaction of grain yield in comparison to plant height explains thus the larger decrease of the accuracy of prediction of GS for grain yield than plant height from one set of evaluation locations to other locations or one testing year to another $\left[\mathrm{CV}_{\mathrm{G} \times \mathrm{L}}\right.$ compared to $\mathrm{CV}_{\mathrm{G}(\mathrm{L})}$ and $\mathrm{CV}_{\mathrm{G} \times \mathrm{Y}}$ compared to $\mathrm{CV}_{\mathrm{G}}(\mathrm{Y})$; Figure 4]. In this context, it has to be considered that testing in only one year limits the accuracy of prediction, indication that in hybrid rye testing for more than one year is of importance to the optimal calibration of genomic selection. This is consistent with observations on limitations set by available data from evaluation locations and testing years for maize made by Kleinknecht et al. [47].

\section{Conclusions}

In hybrid rye, genomic selection is superior to markerassisted selection which generates lower accuracies of prediction which are potentially overestimated. However, high accuracies of prediction are achieved by genomic selection only for candidates closely related to the plant material evaluated in field trials, resulting in a rather pessimistic prognosis for distantly related material. As both, the number of evaluation locations and the number of testing years contribute equally to accuracy field trials for the calibration of genomic selection should be performed in more than one year at several locations.

\section{Additional files}

Additional file 1: Figure S1. Linkage disequilibrium (LD) structure for diversity array technology (DArT) markers. Data was based on 394 and 584 segregating markers within population A and population B, respectively, and 1048 markers across both populations.

Additional file 2: Figure S2. Genotypic values for grain yield, plant height, starch and total pentosan content. Hybrid rye derived from test-crosses of two segregating bi-parental populations was analysed. Data were collected for test-cross progenies from two times $220 \mathrm{~F}_{3: 4}$ lines and their respective parents across nine (for grain yield and plant height) or six (for starch content and total pentosan content) environments, respectively. P1 and P2 refer to the parental lines of population A (Pop-A, Lo115-N x Lo90-N; white), P1 and P3 the parental lines of population B (Pop-B, Lo115-N x Lo117-N; grey).

Additional file 3: Figure S3. Cross-validated standardized accuracies of prediction $\left(r_{g}\right)$ for QTL-based versus random marker-assisted selection. QTL-based marker-assisted selection (MAS-QTL) was performed in comparison to marker-assisted selection performed based on randomly sampled neutral markers (MAS-NEUT). Cross-validation was performed within population $B\left(C_{G}\right.$ Within-Within-Same) for traits grain yield, plant height, starch content, and total pentosan content. QTL mapping based on estimation set data was performed using different limit of detection (LOD) thresholds (numbers below columns), resulting in ranges of median numbers of detected QTL (numbers in the boxes above columns). Analysis was based on 900 DArT markers as described in [5]. 


\section{Abbreviations}

BEK: Beckedorf; BLUEs: Best linear unbiased estimates; CMS: Cytoplasmatic-genic male sterility; CV: Cross-validation; $\mathrm{CV}_{\mathrm{G}}$ : Cross-validation across genotype scheme; $\mathrm{CV}_{\mathrm{G}}$ Across-Across: Cross-validation across genotype scheme in which members of the ES and TS are taken from both populations; $\mathrm{CV}_{\mathrm{G}}$ Across-Within: Cross-validation across genotype scheme in which ES are taken from both populations, while the TS are taken from one population; $\mathrm{CV}_{G}$ Env: Cross-validation across genotype scheme based on different numbers of environments; $\mathrm{CV}_{\mathrm{G}}$ Within-Within: Cross-validation across genotype scheme in which members of the ES and TS are taken from either individual population; $C V_{G}$ Within-Within-Different: $C V_{G}$ Within-Within scheme in which ET and TS are collected from the different population; $\mathrm{CV}_{\mathrm{G}}$ Within-Within-Same: $\mathrm{CV}_{\mathrm{G}}$ Within-Within scheme in which ET and TS originate from the same population $\mathrm{CV}_{\mathrm{G}(L)}$ : Cross-validation across genotype within location scheme in which data for the ES is derived from two randomly selected locations over the two years 2010 and 2011, while the data for the TS is taken either from the same location-year combinations; $\mathrm{CV}_{\mathrm{G} \times \mathrm{L}}$ : Cross-validation across genotype and location scheme in which data for the ES is derived from two randomly selected locations over the two years 2010 and 2011, while the data for the TS is from the remaining four location-year combinations; $\mathrm{CV}_{\mathrm{G}}(n)$ : Cross-validation across genotype within year scheme in which data for the ES was collected from one year, either 2010 or 2011 with data for the TS taken either from the same year; $\mathrm{CV}_{\mathrm{G} \times \mathrm{Y}}$ : Cross-validation across genotype and year scheme in which data for the ES was collected from one year, either 2010 or 2011 with data for the TS taken either from the other year; DArT: Diversity array technology; ES: Estimation set; $\mathrm{G} \times \mathrm{E}$ interaction: Genotype and environment interaction; GS: Genomic selection; HOH: Hohenheim; LD: Linkage disequilibrium; MAS: Marker-assisted selection; MAS-NEUT: Neutral marker-assisted selection; MAS-QTL: Marker-assisted selection based on QTL; PET: Petkus; QTL: Quantitative trait loci; RR-BLUP: Ridge regression best linear unbiased prediction; SNP: Single nucleotide polymorphism; SSR: Simple sequence repeat; TS: Test set; WAL: Walewice; WHO: Wohlde.

\section{Competing interests}

The authors declare that they have no competing interests.

\section{Authors' contributions}

$J C R, T M, P W$ and $Y Z$ outlined the design and supervised the conducting of the study. MG collected the primary data and organized them for biometrical analyses. YW and YZ performed mathematical operations and data analysis and generated the tables and figures. YW and MFM structured the interpretation of results. YW, MFM, and JCR wrote the manuscript. All authors read and approved the final manuscript.

\section{Acknowledgements}

This research was conducted within the project "Erweiterung der genetischen Basis von Hybridroggen für Korn- und Biomasseleistung sowie Trockenheitstoleranz mittels Mehrlinienkartierung und DH-Technik" financially supported by the German Federal Ministry of Food and Agriculture via the "Fachagentur Nachwachsende Rohstoffe e.V.", Gülzow, Germany (Grant ID: 22021711)

\section{Author details}

${ }^{1}$ Leibniz Institute of Plant Breeding and Crop Plant Research (IPK), Gatersleben 06466, Germany. ${ }^{2}$ State Plant Breeding Institute, University of Hohenheim, Stuttgart 70599, Germany. ${ }^{3}$ Syngenta Agro GmbH, Am Technologiepark 1-5, Maintal 63477, Germany. ${ }^{4}$ KWS LOCHOW GMBH, Ferdinand-von-Lochow-Str. 5, 29303 Bergen, Germany.

Received: 27 March 2014 Accepted: 11 June 2014 Published: 4 July 2014

\section{References}

1. Lundqvist A: Self-Incompatibility in rye. Hereditas 1956, 42(3-4):293-348.

2. Voylokov A, Fuong F, Smirnov V: Genetic studies of self-fertility in rye (Secale cereale L.). 1. The identification of genotypes of self-fertile lines for the Sf alleles of self-incompatibility genes. Theor App/ Genet 1993, 87(5):616-618.

3. Geiger H, Schnell F: Cytoplasmic male sterility in rye (Secale cereale L.). Crop Sci 1970, 10(5):590-593.
4. Geiger H, Miedaner T: Hybrid rye and heterosis. In The Genetics and Exploitation of Heterosis in Crops. Edited by JG Pandey S. America, Madison, Wisconsin, USA: Crop Science Society; 1999, 439-450.

5. Miedaner T, Hübner M, Korzun V, Schmiedchen B, Bauer E, Haseneyer G, Wilde P, Reif JC: Genetic architecture of complex agronomic traits examined in two testcross populations of rye (Secale cereale L.). BMC Genomics 2012, 13(1):706.

6. Bernardo R: Molecular markers and selection for complex traits in plants: learning from the last 20 years. Crop Sci 2008, 48(5):1649-1664.

7. Heffner EL, Sorrells ME, Jannink J-L: Genomic selection for crop improvement. Crop Sci 2009, 49(1):1-12

8. Meuwissen THE, Hayes B, Goddard M: Prediction of total genetic value using genome-wide dense marker maps. Genetics 2001, 157(4):1819-1829.

9. Hayes BJ, Bowman PJ, Chamberlain AJ, Goddard ME: Invited review: Genomic selection in dairy cattle: progress and challenges. J Dairy Sci 2009, 92(2):433-443.

10. Albrecht T, Wimmer V, Auinger H-J, Erbe M, Knaak C, Ouzunova M, Simianer $\mathrm{H}$, Schön C-C: Genome-based prediction of testcross values in maize. Theor Appl Genet 2011, 123(2):339-350.

11. Utz HF, Melchinger AE, Schön CC: Bias and sampling error of the estimated proportion of genotypic variance explained by quantitative trait loci determined from experimental data in maize using cross validation and validation with independent samples. Genetics 2000, 154(4):1839-1849.

12. Windhausen VS, Atlin GN, Hickey JM, Crossa J, Jannink JL, Sorrells ME, Raman B, Cairns JE, Tarekegne A, Semagn K: Effectiveness of genomic prediction of maize hybrid performance in different breeding populations and environments. G3 Genes| Genomes| Genetics 2012, 2(11):1427-1436.

13. Zhao Y, Gowda M, Liu W, Würschum T, Maurer HP, Longin FH, Ranc N, Reif JC: Accuracy of genomic selection in European maize elite breeding populations. Theor Appl Genet 2012, 124(4):769-776.

14. Heffner EL, Jannink J-L, Iwata H, Souza E, Sorrells ME: Genomic selection accuracy for grain quality traits in biparental wheat populations. Crop Sci 2011, 51(6):2597-2606

15. Rutkoski J, Benson J, Jia Y, Brown-Guedira G, Jannink J-L, Sorrells M: Evaluation of genomic prediction methods for Fusarium head blight resistance in wheat. Plant Genome 2012, 5(2):51-61.

16. Heslot N, Yang H-P, Sorrells ME, Jannink J-L: Genomic selection in plant breeding: a comparison of models. Crop Sci 2012, 52(1):146-160.

17. Zhong S, Dekkers JC, Fernando RL, Jannink J-L: Factors affecting accuracy from genomic selection in populations derived from multiple inbred lines: a barley case study. Genetics 2009, 182(1):355-364.

18. Habier D, Tetens J, Seefried F-R, Lichtner P, Thaller G: The impact of genetic relationship information on genomic breeding values in German Holstein cattle. Genet Sel Evol 2010, 42(1):5.

19. Daetwyler HD, Pong-Wong R, Villanueva B, Woolliams JA: The impact of genetic architecture on genome-wide evaluation methods. Genetics 2010, 185(3):1021-1031.

20. Zhao $Y$, Zeng J, Fernando R, Reif JC: Genomic prediction of hybrid wheat performance. Crop Sci 2013, 53(3):802-810.

21. Jürgens $H-U$, Jansen $G$, Wegener $C B$ : Characterisation of Several Rye Cultivars with Respect to Arabinoxylans and Extract Viscosity. J Agric Sci 2012, 4(5):1916-9752

22. Gilmour AR, Gogel B, Cullis B, Thompson R: ASRem/ user guide release 3.0. UK: VSN International Ltd, Hemel Hempstead; 2009.

23. Piepho H-P, Möhring J: Computing heritability and selection response from unbalanced plant breeding trials. Genetics 2007, 177(3):1881-1888.

24. Hill W, Robertson A: Linkage disequilibrium in finite populations. Theor Appl Genet 1968, 38(6):226-231.

25. Utz $\mathrm{H}$, Melchinger A: PLABQTL: a program for composite interval mapping of QTL. J Quant Trait Loci 1996, 2(1):1-5.

26. Haley CS, Knott SA: A simple regression method for mapping quantitative trait loci in line crosses using flanking markers. Heredity 1992, 69(4):315-324.

27. Jansen RC, Stam P: High resolution of quantitative traits into multiple loci via interval mapping. Genetics 1994, 136(4):1447-1455.

28. Zeng Z-B: Precision mapping of quantitative trait loci. Genetics 1994 , 136(4):1457-1468.

29. Whittaker IC, Thompson R, DENHAM MC: Marker-assisted selection using ridge regression. Genet Res 2000, 75(02):249-252. 
30. Hofheinz N, Borchardt D, Weissleder K, Frisch M: Genome-based prediction of test cross performance in two subsequent breeding cycles. Theor Appl Genet 2012, 125(8):1639-1645.

31. Dekkers J: Prediction of response to marker-assisted and genomic selection using selection index theory. J Anim Breed Genet 2007, 124(6):331-341.

32. Lande R, Thompson R: Efficiency of marker-assisted selection in the improvement of quantitative traits. Genetics 1990, 124(3):743-756.

33. Guo Z, Tucker DM, Lu J, Kishore V, Gay G: Evaluation of genome-wide selection efficiency in maize nested association mapping populations. TAG Theoretical applied Genetics Theoretische und angewandte Genetik 2012, 124(2):261-275.

34. Heffner EL, Jannink J-L, Sorrells ME: Genomic selection accuracy using multifamily prediction models in a wheat breeding program. Plant Genome 2011, 4(1):65-75.

35. Holland JB: Implementation of molecular markers for quantitative traits in breeding programs-challenges and opportunities. In New Directions for a Diverse Planet: Proceedings for the 4th International Crop Science Congress Regional Institute, Gosford, Australia, www cropscience org au/icsc 2004. 2004.

36. Clark SA, Hickey JM, Daetwyler HD, Van Der Werf JH: The importance of information on relatives for the prediction of genomic breeding values and the implications for the makeup of reference data sets in livestock breeding schemes. Genet Sel Evol 2012, 44:4.

37. Gowda M, Zhao Y, Würschum T, Longin CF, Miedaner T, Ebmeyer E, Schachschneider R, Kazman E, Schacht J, Martinant J: Relatedness severely impacts accuracy of marker-assisted selection for disease resistance in hybrid wheat. Heredity 2013, 112:552-561

38. Habier D, Fernando RL, Dekkers JC: The impact of genetic relationship information on genome-assisted breeding values. Genetics 2007, 177(4):2389-2397.

39. Zhao Y, Gowda M, Longin FH, Würschum T, Ranc N, Reif JC: Impact of selective genotyping in the training population on accuracy and bias of genomic selection. Theor Appl Genet 2012, 125(4):707-713.

40. Riedelsheimer C, Technow F, Melchinger AE: Comparison of whole-genome prediction models for traits with contrasting genetic architecture in a diversity panel of maize inbred lines. BMC Genomics 2012, 13(1):452.

41. Solberg T, Sonesson A, Woolliams J: Genomic selection using different marker types and densities. J Anim Sci 2008, 86(10):2447-2454

42. Haseneyer G, Schmutzer T, Seidel M, Zhou R, Mascher M, Schön CC, Taudien S, Scholz U, Stein N, Mayer KF: From RNA-seq to large-scale genotyping-genomics resources for rye (Secale cereale L.). BMC Plant Biol 2011, 11(1):131.

43. Meuwissen T: Accuracy of breeding values of 'unrelated'individuals predicted by dense SNP genotyping. Genet Sel Evol 2009, 41:35.

44. Ly D, Hamblin M, Rabbi I, Melaku G, Bakare M, Gauch HG, Okechukwu R, Dixon AG, Kulakow P, Jannink J-L: Relatedness and Genotype × Environment Interaction Affect Prediction Accuracies in Genomic Selection: a Study in Cassava. Crop Sci 2013, 53(4):1312-1325.

45. Chee M, Yang R, Hubbell E, Berno A, Huang XC, Stern D, Winkler J, Lockhart DJ, Morris MS, Fodor SP: Accessing genetic information with high-density DNA arrays. Science 1996, 274(5287):610-614.

46. Gagneur J, Elze MC, Tresch A: Selective phenotyping, entropy reduction, and the mastermind game. BMC Bioinformatics 2011, 12(1):406.

47. Kleinknecht K, Möhring J, Singh K, Zaidi P, Atlin G, Piepho H: Comparison of the Performance of Best Linear Unbiased Estimation and Best Linear Unbiased Prediction of Genotype Effects from Zoned Indian Maize Data. Crop Sci 2013, 53(4):1384-1391.

doi:10.1186/1471-2164-15-556

Cite this article as: Wang et al:: The accuracy of prediction of genomic selection in elite hybrid rye populations surpasses the accuracy of marker-assisted selection and is equally augmented by multiple field evaluation locations and test years. BMC Genomics 2014 15:556.

\section{Submit your next manuscript to BioMed Central and take full advantage of:}

- Convenient online submission

- Thorough peer review

- No space constraints or color figure charges

- Immediate publication on acceptance

- Inclusion in PubMed, CAS, Scopus and Google Scholar

- Research which is freely available for redistribution

Submit your manuscript at www.biomedcentral.com/submit
() Biomed Central 